\title{
A Tuning Method for Electrically Compensated Ion Cyclotron Resonance Mass Spectrometer Traps
}

\author{
Adam M. Brustkern, Don L. Rempel, and Michael L. Gross \\ Department of Chemistry, Washington University in St. Louis, St. Louis, Missouri, USA
}

\begin{abstract}
We describe a method for tuning electrically compensated ion cyclotron resonance (ICR) traps by tracking the observed cyclotron frequency of an ion cloud at different oscillation mode amplitudes. Although we have used this method to tune the compensation voltages of a custom-built electrically compensated trap, the approach is applicable to other designs that incorporate electrical compensation. To evaluate the effectiveness of tuning, we examined the frequency shift as a function of cyclotron orbit size at different z-mode oscillation amplitudes. The cyclotron frequencies varied initially by $\sim 12 \mathrm{ppm}$ for ions with low z-mode oscillation amplitudes compared with those with high z-mode amplitudes. This frequency difference decreased to $\sim 1$ ppm by one iteration of trap tuning. (J Am Soc Mass Spectrom 2010, 21, 451-454) () 2010 Published by Elsevier Inc. on behalf of American Society for Mass Spectrometry
\end{abstract}

$\mathrm{F}$ ourier transform ion cyclotron resonance mass spectrometry (FT-ICR MS) offers ultra high mass resolving powers and accurate mass measurements that surpass those of other methods. The trap serves as the heart of the instrument, allowing ions to be stored, fragmented, and analyzed. Ion motion in traps is confined in the radial direction by a strong homogeneous magnetic field, and in the axial direction by a three-dimensional quadrupolar electrostatic trapping well. As a consequence of the finite dimensions of the trapping-plate electrodes, the electrostatic trapping potential within the trap is only approximately quadrupolar. The resulting outwardly directed radial electric field $\left(E_{r}\right)$ opposes the effect of the magnetic field and causes a shift in the observed cyclotron frequency of the ions and a procession of the ion clouds' guiding center (magnetron motion).

A problem arises because the effect of the $E_{r}$ field is not constant, causing the observed cyclotron frequency to depend on ion-mode amplitudes within the trap. Among the efforts to design a trap in which the ion cyclotron frequency is independent of its mode amplitudes are those that attempt to eliminate the radial electric field by creating an electrostatic well shape that approximates a particle-in-a-box [1-9] $\left(E_{r}=0\right)$. Other strategies seek a design that more closely approximates the desired three-dimensional quadrupolar trapping potential [10-15] $\left(E_{r} / r=\right.$ constant $)$. The outcomes are traps with complex electrode shapes that follow the equipotential lines of a perfect, three-dimensional quadrupolar potential (i.e., a hyperbolic trap) and others

Address reprint requests to Dr. M. L. Gross, Department of Chemistry, Washington University, Campus Box 1134, One Brookings Drive, St. Louis, MO, 63130, USA. E-mail: mgross@wustl.edu with electrodes segmented into many parts to which individual compensation voltages are applied to shape the trapping potential.

Our solution to this problem is an electrically compensated trap [10] whose implementation requires that correct voltages be applied to the electrodes to reduce the sensitivity of cyclotron frequency as a function of mode amplitudes (i.e., $d \omega / d A_{z, r} \rightarrow 0$ ). Despite our best efforts, the compensation voltages calculated by theory do not afford optimum performance, although we did find an improvement in performance over an unmodified trap [10, 11], as did others [1-9, 12-15] who used theoretical designs.

Inability to manufacture the trap in accordance with theory and variations of contact potentials of the electrode surfaces may account for deviations from theory. Further, tuning the compensation voltages is necessary to correct for potential variations caused by electrode aging. Tuning frequency depends on instrument use, baking, and vacuum cycles. Given the lack of concordance between theory and experiment, we needed an effective strategy for tuning the compensation voltages to realize the trap's full potential. Tuning methods that rely on changing the compensation voltages in response to observed peak shape, akin to tuning a "beam" mass spectrometer, are impractical because implementing them on an FT-ICR instrument would be highly timeconsuming, owing to the very large number of possible voltage combinations and the long observation times $(>100 \mathrm{~s})$ that are required to yield the highest performance. For example, if a range of $1 \mathrm{~V}$ for each of the three compensation voltages is investigated using steps of 50 $\mathrm{mV}, 8000$ different combinations result. When transient acquisition times are $100 \mathrm{~s}$, the total time to investigate these possibilities is over nine days. Using the new tuning
(C) 2010 Published by Elsevier Inc. on behalf of American Society for Mass Spectrometry. 1044-0305/10/\$32.00

doi:10.1016/j.jasms.2009.11.010
Published online December 11, 2009

Received September 1, 2009

Revised November 12, 2009

Accepted November 24, 2009 


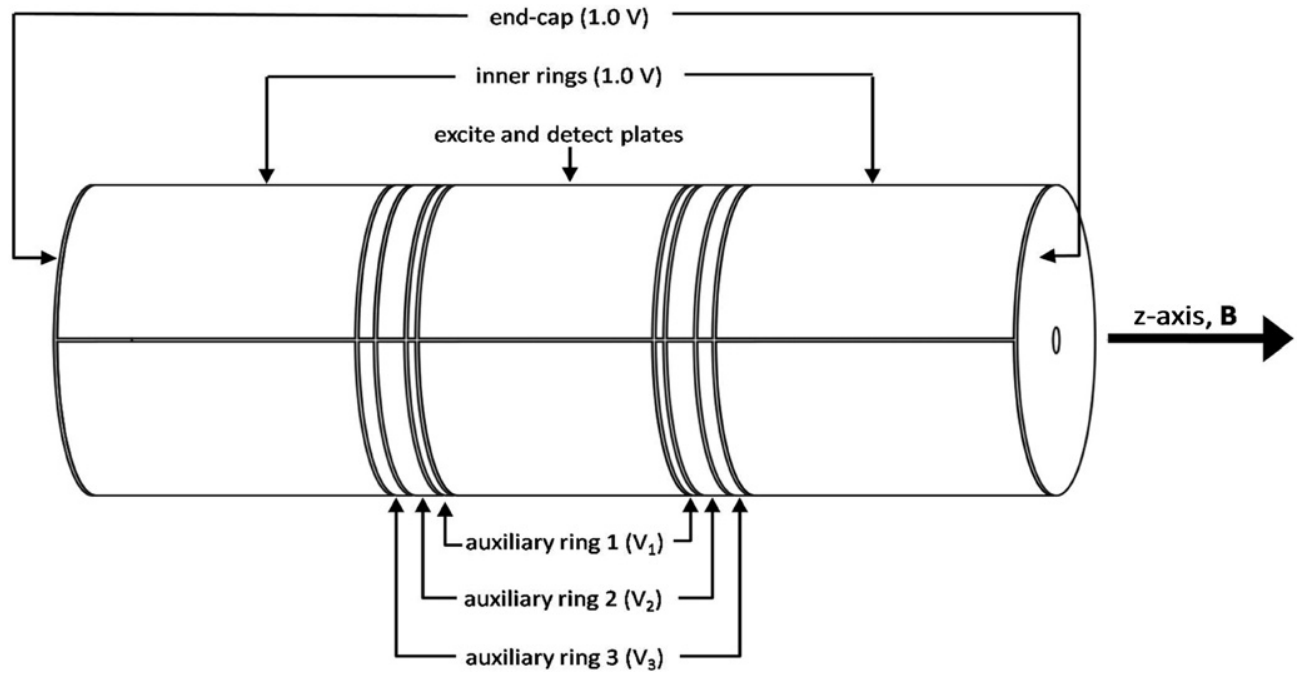

Figure 1. Diagram of the compensated trap, installed on a Varian (IonSpec) ProMALDI FTMS system, showing the positions of the three auxiliary ring pairs.

method, we were able to accomplish it in a single day. Another difficulty encountered using peak shape for tuning is that the underlying frequency information can be obscured by phase locking and this makes deduction of the shape of the frequency surface impossible.

Here we describe a tuning method based on the experimentally observed frequency centroids produced from ion clouds of differing mode amplitudes to probe the frequency surface of a compensated trap. The approach has advantages because frequency centroids of a collection of ions are essentially unaffected by phase locking. The tuning strategy is applicable to most, if not all, electrically compensated ICR traps.

\section{Experimental}

The analyte, $[\mathrm{Arg}]^{8}$-vasopressin, from Sigma Aldrich (St. Louis, MO, USA), was mixed with 2,5-dihydroxybenzoic acid at a ratio of 1:3000 before spotting on a standard 192-spot stainless steel sample plate. Spectra were acquired with a 7.0-Tesla IonSpec ProMALDI FTMS (Lake Forest, CA, USA), equipped with an external hexapole for accumulation and collisional cooling of ions from multiple laser shots. The ions were subsequently transferred to the custom-built ICR trap, shown in Figure 1, via a quadrupolar ion-transfer guide. The end-cap electrodes and the inner rings were at $1.0 \mathrm{~V}$ to provide an electrostatic trapping well. The inner rings that supply the electrostatic trapping well should not be confused with the auxiliary rings to which the compensation voltages $\left(\mathrm{V}_{1}, \mathrm{~V}_{2}\right.$, and $\left.\mathrm{V}_{3}\right)$ are applied. The auxiliary and inner rings of the modified trap were segmented and capacitively coupled to the excite plates to minimize axial ejection during excitation. The amplitude of the arbitrary waveform (ARB) used for excitation was varied to control the cyclotron orbit size of the ion cloud. The post-excitation radius of a cloud excited with a 275
$\mathrm{V}$ ARB waveform is $\sim 50 \%$ of the trap radius and varies almost linearly with the excitation amplitude.

The z-mode amplitude distribution of an ion cloud was manipulated by collisionally cooling the ions with three $10 \mathrm{~ms}$ pulses of $\mathrm{N}_{2}$ gas, followed by z-mode re-excitation, driven by applying $+35 \mathrm{~V}$ to the entrance end-cap and $-35 \mathrm{~V}$ to the filament side end-cap; the duration of the end-cap pulse controlled the extent of re-excitation in the z-direction. Ion clouds re-excited for longer times were considered to have average z-mode amplitudes that were larger than those that had short or no z-mode re-excitation. Assuming that the center of the ion cloud following nitrogen pulsing is at the trap center, we calculated that the z-mode amplitude of an ion cloud excited by a $300 \mu$ s pulse was $\sim 2.6 \mathrm{~cm}$.

Digitized time domain signals of $1024 \mathrm{~K}$ data points were acquired in the narrowband mode for vasopressin at an $\mathrm{ADC}$ rate of $10 \mathrm{kHz}$, corresponding to an acquisition time of $\sim 105 \mathrm{~s}$ per detection event. Following acquisition, the data were imported into a FORTRAN program for calculation of the complex FFT, magnitude-mode frequency centroid, complex area, and mass resolving power.

\section{Results and Discussion}

Our first goal is to assess the effectiveness of a compensation scheme. The appropriate indicator of compensation efficacy is the slope (flatness) of a curve produced when frequency is plotted versus cyclotron orbit size at different z-mode oscillation amplitudes. Ideally these curves would be straight lines on top of one another with zero slopes. We invoke the idea of a "frequency surface," which describes the cyclotron frequency of an ion as a function of both its z-mode oscillation amplitude and its cyclotron orbit size. A frequency surface is analogous to a topological map, with frequency replac- 
ing elevation. Examination of the surface allows for evaluation of the cyclotron frequency shift as a function of mode amplitudes. Ideally, the frequencies would not vary as a function of $\mathrm{z}$ - or cyclotron-mode amplitude (i.e., the frequency surface would be flat).

Tuning was accomplished by acquiring spectra using different combinations of cyclotron orbit size and zmode oscillation amplitude for a given set of compensation voltages. This approach samples the "frequency surface" for that set of compensation voltages. For example, the cyclotron orbit size was varied by using an ARB waveform amplitude of either $150 \mathrm{~V}_{(b-p)}$ or 325 $\mathrm{V}_{(b-p)}$, corresponding to post-excitation cyclotron orbits of $\sim 30$ and $60 \%$ the radius of the trap, respectively. The z-mode oscillation amplitude was controlled by changing the duration of the $\mathrm{z}$-mode excitation pulse from 100 to $300 \mu \mathrm{s}$. This approximately corresponds to a z-mode amplitude of 0.8 and $2.6 \mathrm{~cm}$ for the "cool" and "hot" ion clouds, respectively. The change in magnitude-mode frequency centroids across the surface in a particular direction, $\Delta f_{i j}$ (where " $i$ " represents a direction and " $j$ " a tuning state) is the difference between the ion frequencies at low cyclotron orbit size and low z-mode oscillation amplitude from those observed at any point in the tuning. The process, using four points, is represented in Figure 2. It is important to have a reference frequency within each set because the depth of the electrostatic trapping well varies as the compensation voltages are adjusted (this is not necessarily so if the trap were designed to orthogonalize the well depth and compensation voltages). Here, we chose the frequency at low cyclotron and low z-mode oscillation amplitudes as our reference point. Once the $\Delta f$ values for the initial compensation voltages were collected, the voltage on one compensation electrode pair was changed, and the process repeated until a set of $\Delta f$ values was obtained for a step in compensation voltage for each auxiliary ring pair. After the four sets of $\Delta f$ values were obtained,

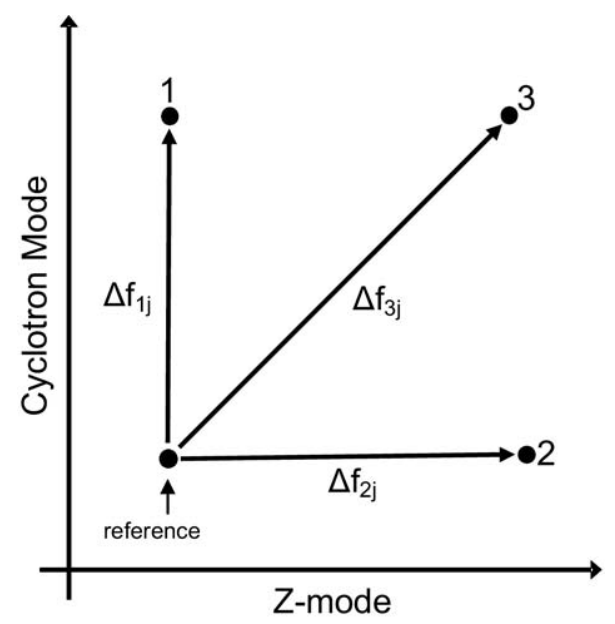

Figure 2. Tuning the compensated trap by determining the sensitivity of cyclotron frequency to z-mode or cyclotron-mode amplitudes. the effect of each voltage change on the frequency surface was calculated by subtracting the $\Delta f_{i}$ values obtained when using the starting set of compensation voltages from the $\Delta f_{i j}$ values obtained when the voltage on an auxiliary ring pair had been changed. This difference was normalized by the change in voltage applied (these are the $\Delta \Delta f_{i j}$ values). These values are a way to convert a voltage change $(\Delta V)$ into the resulting frequency change $(\Delta f)$.

$$
\begin{aligned}
& \Delta \Delta f_{11} \Delta V_{1}+\Delta \Delta f_{12} \Delta V_{2}+\Delta \Delta f_{13} \Delta V_{3}+\ldots=\Delta f_{1} \\
& \Delta \Delta f_{21} \Delta V_{1}+\Delta \Delta f_{22} \Delta V_{2}+\Delta \Delta f_{23} \Delta V_{3}+\ldots=\Delta f_{2} \\
& \Delta \Delta f_{31} \Delta V_{1}+\Delta \Delta f_{32} \Delta V_{2}+\Delta \Delta f_{33} \Delta V_{3}+\ldots=\Delta f_{3}
\end{aligned}
$$

For an arbitrary number of compensation electrodes, the system of equations that give $\Delta f$ as a function of $\Delta V$ can be written as eq 1 . The $\Delta V$ values can be viewed as representing the difference between the desired voltages that give a flat surface and the unoptimized starting voltages resulting in the observed set of $\Delta f$ values. In this way, the $\Delta V$ values are corrections that

$$
\underbrace{\left(\begin{array}{lll}
\Delta \Delta f_{11} & \Delta \Delta f_{12} & \Delta \Delta f_{13} \\
\Delta \Delta f_{21} & \Delta \Delta f_{22} & \Delta \Delta f_{23} \\
\Delta \Delta f_{31} & \Delta \Delta f_{32} & \Delta \Delta f_{33}
\end{array}\right)}_{\Delta \Delta f}(\underbrace{\left(\begin{array}{c}
\Delta V_{1} \\
\Delta V_{2} \\
\Delta V_{3}
\end{array}\right)}_{\Delta V}=\underbrace{\left(\begin{array}{c}
\Delta f_{1} \\
\Delta f_{2} \\
\Delta f_{3}
\end{array}\right)}_{\Delta f}
$$

must be removed from the starting set to move closer to the ideal compensation voltages. In general, the soughtafter $\Delta V$ values minimize the residuals between the calculated and the experimental $\Delta f$ values and are found by utilizing the derivatives of frequency change $(\Delta \Delta f$ values) as a function of voltages applied to the compensation ring pairs. In our case, the number of $\Delta f$ values equaled the number of available voltages that could be changed. This fact allowed us to condense the above system of equations into a square matrix equation (eq 2 ). The process is analogous to the Newton method used to find the zero of a function. As with most search methods, there is a possibility of falling into a local minimum. This prospect is not an obstacle as long as the voltages found at the local minimum result in a flat frequency surface.

This system was easily solved by finding the inverse of the $\Delta \Delta f$ matrix and left multiplying it on both sides of the equation (eq 3). What remained after simplification is an expression for the correction voltages, $\Delta V$. The $\Delta V$ values were then subtracted from the initial set of compensation voltages, moving from a less to a more ideal tuning situation.

$$
\begin{aligned}
\Delta \Delta f \Delta V & =\Delta f \\
\Delta \Delta f^{-1} \Delta \Delta f \Delta V & =\Delta \Delta f^{-1} \Delta f \\
\Delta V & =\Delta \Delta f^{-1} \Delta f
\end{aligned}
$$




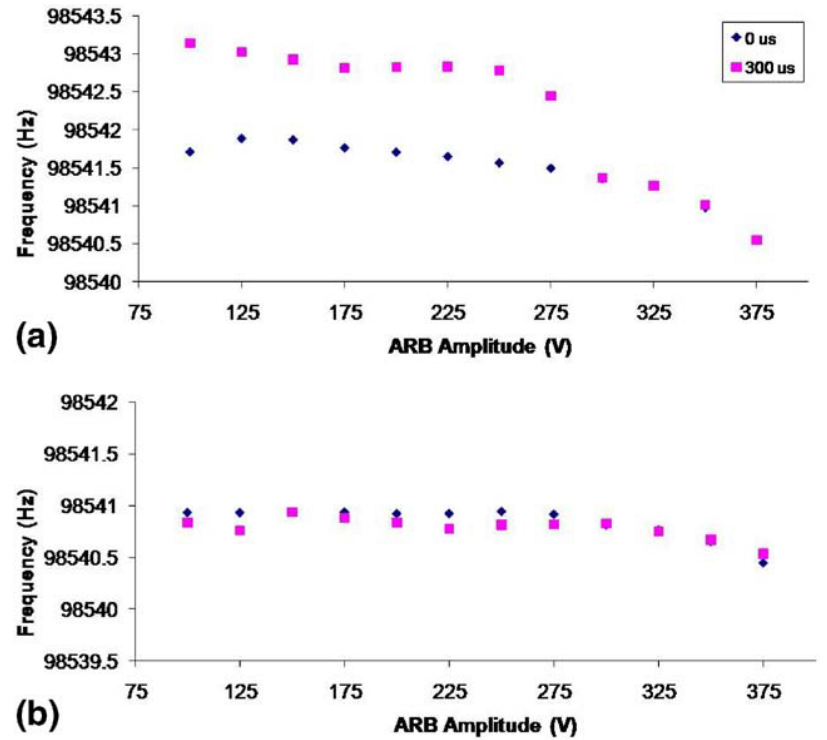

Figure 3. Variation in cyclotron frequency (at $\mathrm{m} / \mathrm{z} 1084$ ) as a function of cyclotron orbit size and z-mode oscillation amplitude before (a) and after tuning (b). The frequency centroids produced from the high $(300 \mu \mathrm{s})$ and low $(0 \mu \mathrm{s}) \mathrm{z}$-mode amplitude spectra are represented as squares and diamonds, respectively.

Cyclotron orbit scans that resulted from application of the starting or theoretical compensation voltages (Figure 3a) show that the cyclotron frequency varies as a function of cyclotron orbit size (mode amplitude). More importantly, the cyclotron frequency depends strongly on the z-mode amplitude, as for all non-ideal traps. The sudden shift in frequency of the high z-mode amplitude cloud results because the portion of the ion cloud responsible for the detected centroids changes, not because the frequency surface itself is suddenly flat. At ARB amplitudes higher than $275 \mathrm{~V}$, the highfrequency, low-resolving-power portion of the ion cloud is no longer detectable above noise and, therefore, it does not add to the measured centroids. One iteration of tuning produced a nearly optimized situation. Not only was the frequency shift as a function of cyclotron mode oscillation amplitude greatly decreased (Figure $3 b$ ), but also the frequency shift observed from low to high z-mode oscillation amplitude was reduced by an order of magnitude (average of $\sim 12$ to $\sim 1 \mathrm{ppm}$ ) for protonated vasopressin of $\mathrm{m} / \mathrm{z} 1084.5$, a realistic test.

\section{Conclusions}

Electrical trap compensation is effective in reducing frequency shift as a function of ion mode amplitudes, provided that the compensation voltages supplied to the trap are correct. We describe a new strategy for tuning based on measuring the variation of the ob- served cyclotron frequency of an ion cloud with different mode-amplitude distributions. We are able to relate the changes in frequency that occur as the compensation voltages are intentionally varied to a voltage change that accounts for the initially observed frequency shifts. The tuning method is effective in reducing the frequency shift as a function of mode amplitude, and we propose that our method is generally applicable to most, if not all, compensated traps that employ electrical compensation.

\section{Acknowledgments}

The authors acknowledge support for this research by the National Centers for Research Resources of the NIH (grant 2P41RR000954). They thank Dr. Robert McIver for his help in the implementation of the compensated trap.

\section{References}

1. Bruce, J. E.; Anderson, G. A.; Lin, C.-Y.; Gorshkov, M.; Rockwood, A. L.; Smith, R. D. A Novel High-Performance Fourier Transform Ion Cyclotron Resonance Cell for Improved Biopolymer Characterization. J. Mass Spectrom. 2000, 35, 85-94.

2. Hanson, C. D.; Castro, M. E.; Kerley, E. L.; Russell, D. H. Field-Corrected Ion Cell for Ion Cyclotron Resonance. Anal. Chem. 1990, 62, 520-526.

3. Hunter, R. L.; Sherman, M. G.; McIver, R. T., Jr. An Elongated Trapped-Ion Cell for Ion Cyclotron Resonance Mass Spectrometry with a Superconducting Magnet. Int. J. Mass Spectrom. Ion Phys. 1983, 50, 259-274.

4. Kaiser, N. K.; Bruce, J. E. Observation of Increased Ion Cyclotron Resonance Signal Duration through Electric Field Perturbations. Anal. Chem. 2005, 77, 5973-5981.

5. Kaiser, N. K.; Bruce, J. E. Reduction of Ion Magnetron Motion and Space Charge Using Radial Electric Field Modulation. Int. J. Mass Spectrom. 2007, 265, 271-280.

6. Naito, Y.; Fujiwara, M.; Inoue, M. Improvement of the Electric Field in the Cylindrical Trapped-Ion Cell. Int. J. Mass Spectrom. Ion Processes 1992, 120, 179-192.

7. Vartanian, V. H.; Hadjarab, F.; Laude, D. A. Open Cell Analog of the Screened Trapped-Ion Cell Using Compensation Electrodes for Fourier Transform Ion Cyclotron Resonance Mass Spectrometry. Int. J. Mass Spectrom. Ion Processes 1995, 151, 175-187.

8. Vartanian, V. H.; Laude, D. A. Optimization of a Fixed-Volume Open Geometry Trapped Ion Cell for Fourier Transform Ion Cyclotron Mass Spectrometry. Int. J. Mass Spectrom. Ion Processes 1995, 141, 189-200.

9. Wang, M.; Marshall, A. G. A "Screened" Electrostatic Ion Trap for Enhanced Mass Resolution, Mass Accuracy, Reproducibility, and Upper Mass Limit in Fourier-Transform Ion Cyclotron Resonance Mass Spectrometry. Anal. Chem. 1989, 61, 1288-1293.

10. Brustkern, A. M.; Rempel, D. L.; Gross, M. L. An Electrically Compensated Trap Designed to Eighth Order for FT-ICR Mass Spectrometry. J. Am. Soc. Mass Spectrom. 2008, 19, 1281-1285.

11. Gooden, J. K.; Rempel, D. L.; Gross, M. L. Evaluation of Different Combinations of Gated Trapping, rf-Only Mode and Trap Compensation for In-Field MALDI Fourier Transform Mass Spectrometry. J. Am. Soc. Mass Spectrom. 2004, 15, 1109-1115.

12. Jackson, G. S.; White, F. M.; Guan, S.; Marshall, A. G. Matrix-Shimmed Ion Cyclotron Resonance Ion Trap Simultaneously Optimized for Excitation, Detection, Quadrupolar Axialization, and Trapping. J. Am. Soc. Mass Spectrom. 1999, 10, 759-769.

13. Tolmachev, A. V.; Robinson, E. W.; Wu, S.; Kang, H.; Lourette, N. M.; Pasa-Tolic, L.; Smith, R. D. Trapped-Ion Cell with Improved DC Potential Harmonicity for FT-ICR MS. J. Am. Soc. Mass Spectrom. 2008, $19,586-597$.

14. Van Dyck, R. S. Jr.; Schwinberg, P. B. Preliminary Proton/Electron Mass Ratio Using a Compensated Quadring Penning Trap. Phys. Rev. Lett. 1981, 47, 395-398.

15. Van Dyck, R. S. Jr.; Wineland, D. J.; Ekstrom, P. A.; Dehmelt, H. G. High Mass Resolution with a New Variable Anharmonicity Penning Trap. Appl. Phys. Lett. 1976, 28, 446-448. 